\title{
Detección de anticuerpos contra Trypanosoma cruzi en caninos de Costa Rica
}

\section{Detection of antibodies against Trypanosoma cruzi in dogs from Costa Rica}

\author{
Marta C. Bonilla ${ }^{1 凶}$, Marco Vinicio Herrero-Acosta ${ }^{1}$, Andrea Urbina-Villalobos ${ }^{2}$, Gaby Dolz $^{1}$
}

1 Laboratorio de Docencia e Investigación en Medicina Poblacional, Programa MEDPOB, Escuela de Medicina Veterinaria, Universidad Nacional, Heredia, Costa Rica. E-mails: mabongo27@gmail.com, gaby.dolz.wiedner@una.cr, herrero1958@hotmail.com

2 Laboratorio de Zoonosis, Escuela de Medicina Veterinaria, Universidad Nacional, Heredia, Costa Rica. E-mail: andreaurbina3@gmail.com

Recibido: 27 de abril de 2018. Corregido: 21 de junio de 2018. Aceptado: 22 de junio de 2018.

Resumen: La enfermedad de Chagas es una enfermedad vectorial zoonótica ocasionada por Trypanosoma cruzi y transmitida, principalmente, por Triatoma dimidata, en Costa Rica. El parásito puede infectar a humanos, animales domésticos y silvestres. En el país, se habían realizado estudios sobre la seroprevalencia del agente en caninos en 12 de un total de 82 cantones y se encontró en todos ellos perros infectados con T. cruzi. El objetivo de la presente investigación fue determinar la seroprevalencia de T. cruzi en perros de 28 cantones costarricenses y establecer si los caninos seropositivos se presentaban únicamente en cantones donde se había reportado el vector o el parásito en perros, o si se presentaban casos en otros cantones del país aún no investigados. Se analizaron 427 sueros caninos provenientes de 28 cantones ( 23 no investigados con anterioridad), mediante las pruebas de hemaglutinación indirecta (HAI) e inmunofluorescencia indirecta (IFI). La seroprevalencia determinada en los 28 cantones estudiados fue $1.17 \%$. Un total de 5 caninos en 4 cantones (Mora, San Rafael de Heredia, Liberia y Golfito) resultó positivo. Por primera vez, se estableció la presencia de caninos seropositivos de T. cruzi en 3 cantones no reportados antes (Mora, Liberia y Golfito). Se deben ampliar estudios en los cantones con casos positivos, para conocer las características epidemiológicas que promuevan la aparición de infecciones por T. cruzi. Se recomienda informar a los médicos veterinarios que tomen en cuenta la enfermedad de Chagas como un diagnóstico diferencial en perros, especialmente, en los cantones donde se ha reportado la presencia del agente y continuar con estudios sistemáticos, en el nivel de comunidades y cantones, para determinar si la infección se encuentra distribuida en todo el país.

Palabras claves: seroprevalencia, anticuerpos, hemaglutinación indirecta (HAI), inmunofluorescencia indirecta (IFI), cantones.

Abstract: Chagas disease is a zoonotic vector infection caused by Trypanosoma cruzi, and transmitted mainly by Triatoma dimidata in Costa Rica. The parasite infects humans, domestic and wild animals. Serological studies in dogs had been carried out in 12 of the 82 counties of Costa Rica, and dogs infected with T. cruzi were found in all the counties studied. The objective of the present research was to determine the seroprevalence of T. cruzi in dogs of 28 counties of Costa Rica and to establish if the seropositive dogs were presented in counties, where the vector or parasite had been previously reported in dogs, or if they were cases in other counties of the country not yet investigated. A total of 427 canine sera from 28 counties ( 23 not previously

凶

Autor de correspondencia: Marta C. Bonilla, mabongo27@gmail.com 
investigated) were analyzed by indirect hemagglutination (IHA) and indirect immunofluorescence assay (IFA). The seroprevalence determined in the 28 counties studied was $1.17 \%$. A total of 5 canines in 4 counties (Mora, San Rafael de Heredia, Liberia and Golfito) were positive. The presence of T. cruzi seropositive canines was established for the first time in 3 counties not reported before (Mora, Liberia and Golfito). More studies should be done in the counties with positive cases to determine the epidemiological characteristics that promote the appearance of $T$. cruzi infections. It is recommended to inform veterinarians about Chagas disease so they can consider it as a differential diagnosis in dogs, especially in counties where the presence of the agent has been reported, and to continue with systematic studies in communities and counties, to determine if the infection is distributed throughout the country.

Keywords: seroprevalence, antibodies, indirect hemagglutination (IHA), indirect immunofluorescence (IFA), counties.

\section{Introducción}

La enfermedad de Chagas o tripanosomiasis americana es una enfermedad zoonótica producida por el protozoario Trypanosoma cruzi, el cual puede infectar a humanos, animales domésticos y silvestres (Galvao \& Justi 2015). Este parásito es transmitido por insectos hematófagos que pertenecen al orden Hemiptera, familia Reduviidae y subfamilia Triatominae (Zeledón et al. 2016). En Costa Rica, el principal vector de la enfermedad de Chagas es el Triatoma dimidiata (Zeledón 1981). Esta enfermedad es considerada uno de los padecimientos parasitarios más importantes en América y de gran importancia para la salud pública. Se estima que alrededor de 8 millones de personas en América Latina se encuentran infectadas por T. cruzi y 110 millones están en riesgo de contraer la infección (Barbabosa-Pliego et al. 2011; Greene 2012). La Organización Mundial de la Salud (OMS) reporta 21 países de América Latina como endémicos para la enfermedad de Chagas y entre ellos se encuentra Costa Rica (Organización Mundial de la Salud 2017).

Los estudios serológicos en caninos son pocos y, generalmente, se realizan en localidades puntuales donde se sospecha de infecciones caninas y se desea conocer la situación actual de la región. Estudios serológicos en Estados Unidos encontraron, en el estado de Texas, rangos entre 3.6 y 57.6 \% de perros infectados; además, se ha reportado la presencia del protozoario en perros en los estados de Louisiana, Oklahoma, Tennessee y Virginia (Meyers et al. 2017). En México, se han determinado seroprevalencias en rangos desde $0.34 \%$ hasta $34 \%$, dependiendo de la zona en estudio (Arce-Fonseca et al. 2017), mientras que en zonas rurales de Panamá y Brasil se reportan un $17.6 \%$ y un $28 \%$ de caninos seropositivos a T. cruzi, respectivamente (Saldaña et al. 2015; Dias-Pérez et al. 2016).

Los estudios que se han realizado en Costa Rica con caninos han sido puntuales, sobre todo en zonas donde previamente se había reportado la presencia del vector o la aparición de casos de tripanosomiasis canina. De esta manera, se ha estudiado la presencia de T. cruzi en caninos en 12 cantones del país, en los que se había reportado la presencia de Triatoma dimidiata; en ellos. se encontraron caninos seropositivos y los porcentajes de positividad fluctuaron entre 6.7 \% y 27.7 \% (Montenegro et al. 2002; Villegas 2008; Lizundia et al. 2014).

En Costa Rica se registró, por primera vez, la enfermedad aguda en 2 personas, en 1941. Desde entonces, se reportaron casos en 5 de las 7 provincias de Costa Rica: San José, Heredia, Alajuela, Puntarenas y 
Guanacaste (Pérez et al. 1984; Matamoros 2002; Ministerio de Salud 2015). A partir del 2003, se inicia con el tamizaje para la enfermedad de Chagas del $100 \%$ de las donaciones sanguíneas del país. Este monitoreo estableció un $0.07 \%$ de donadores de sangre infectados con T. cruzi, en el 2013 (CamposFuentes \& Calvo-Fonseca 2013).

Para el diagnóstico de T. cruzi en humanos y caninos, se recomienda, sobre todo, la utilización de pruebas serológicas, ya que los anticuerpos están presentes toda la vida o durante el tiempo de infección (Tenney et al.2014). Las técnicas más utilizadas son el inmunoensayo enzimático absorbente (ELISA), la inmunofluorescencia indirecta (IFI) y la hemaglutinación indirecta (HAI). Se recomienda la utilización de al menos 2 técnicas serológicas para confirmar los resultados positivos (Nieto et al. 2009).

El perro se considera el hospedador doméstico más importante de esta enfermedad zoonótica. En condiciones naturales, los perros son infectados con mayor frecuencia que los humanos, por lo que pueden ser utilizados como centinelas para estimar las infecciones en humanos (Barbabosa-Pliego et al. 2011; Castillo-Neyra et al. 2015).

En vista de la escasa información actualizada sobre la tripanosomiasis canina en Costa Rica, la presente investigación pretendió determinar la seroprevalencia de T. cruzi en perros de 28 cantones de Costa Rica y establecer la presencia de canes seropositivos en cantones del país, donde no se habían realizado estudios en estos animales o no se había reportado la presencia de triatominos.

\section{Metodología}

\section{Tipo de estudio}

Se realizó un estudio observacional, transversal y descriptivo, para determinar la presencia de anticuerpos contra T. cruzi, en 427 caninos de Costa Rica.

\section{Procedencia, tipo y tamaño de la muestra}

Las muestras que se utilizaron para el estudio procedían de un banco de sueros de 427 caninos, los cuales fueron recolectados entre junio del 2011 y mayo del 2014, en el marco del proyecto "Diagnóstico molecular de agentes infecciosos en garrapatas y de animales domésticos de distintas áreas recreativas de Costa Rica" (Barrantes-González et al. 2016a), con el objetivo de detectar agentes infecciosos transmitidos por vectores y que se encontraban debidamente almacenados a $-20{ }^{\circ} \mathrm{C}$, en la Escuela de Medicina Veterinaria de la Universidad Nacional.

El cálculo de la muestra se basó en una población total de 1939142 perros en el país, ya que estudios determinaron que, en promedio, existen 1.6 perros por cada vivienda y para el 2011 había un total de 1211964 viviendas ocupadas en Costa Rica, de las cuales se encontraban el 33.1 \% en San José, 19.5 \% en Alajuela, 10.8 \% en Cartago, 10.1 \% en Heredia, 9.8 \% en Puntarenas, $9.0 \%$ en Limón y $7.6 \%$ en Guanacaste (List 2009; Fuprovi 2012; INEC 2012; Sociedad Mundial para la Protección Animal 2012). El cálculo se realizó por medio del programa WinEpiscope 2.0, con una prevalencia mínima estimada del $6 \%$ para T. cruzi (Montenegro et al. 2002; Villegas 2008; Lizundia et al. 2014) y con un 
nivel de confianza del $95 \%$, con lo cual se obtuvo un tamaño de muestra estimado de 300 animales. Sin embargo, para darle más validez a la muestra, se analizaron un total de 427 sueros de caninos, 158 de San José (37 \%), 35 de Alajuela (8.2 \%), 26 de Cartago (6.1\%), 27 de Heredia (6.3\%), 60 de Puntarenas (14.0\%), 44 de Limón (10.3 \%) y 77 de Guanacaste (18 \%).

Los sueros caninos que se analizaron provenían de 28 cantones: Central de Alajuela (19 sueros), San Carlos (16), Central de Cartago (18), El Guarco (4), La Unión (2), Oreamuno (2), Cañas (42), Liberia (35), Central de Heredia (6), San Pablo (2), San Rafael (16), Santo Domingo (3), Central de Limón (22), Pococí (22), Garabito (28), Golfito (32), Alajuelita (3), Aserrí (17), Central de San José (47), Curridabat (1), Desamparados (45), Escazú (11), Montes de Oca (2), Mora (23), Moravia (1), Santa Ana (1), Tibás (5), Vázquez de Coronado (2).

En un total de 21 cantones, se había reportado la presencia del vector, mientras que en 7 (Vázquez de Coronado, Cartago Centro, Oreamuno, El Guarco, Golfito, Garabito y Limón Centro) no se encontraron registros de la presencia del vector (Zeledón 1981; Instituto Nacional de Biodiversidad 2017).

\section{Recolección de datos}

De cada suero canino se tenía registrada la información del propietario (nombre, dirección, teléfono y correo electrónico), datos del canino (nombre, raza, sexo, edad, si vivía dentro del hogar y con otros animales), signos clínicos observados por los propietarios en el pasado (anorexia, membranas mucosas pálidas, intolerancia al ejercicio, letargia, desmayos, ascitis, arritmias, dificultad respiratoria, entre otros), como también, los resultados de la revisión clínica el día de la toma de la muestra (condición corporal, actitud, color de las membranas mucosas, tiempo de llenado capilar, temperatura, presencia de ectoparásitos y signos clínicos observados en el momento del examen clínico), información que estaba guardada en una base de datos.

De los 427 caninos analizados en este estudio, 219 fueron hembras y 208 machos; de estos, 187 fueron cachorros (1-12 meses), 201 adultos (2-7 años) y 39 caninos geriátricos (8-15 años). La mayoría (234) fueron perros sin raza definida (SRD) y 193 de raza (american pitbull terrier, american staffordshire terrier, beagle, bichón habanero, bichón maltés, bóxer, bull terrier inglés, bulldog inglés, caniche, chihuahua, cocker spaniel inglés, collie, dálmata, dóberman, golden retriever, husky siberiano, labrador retriever, pastor alemán, pequinés, pinscher ,miniatura, pomerano, rottweiler, samoyedo, schnauzer miniatura, shih tzu, weimaraner, west highland white terrier y whippet).

\section{Análisis serológico}

Todos los sueros se sometieron a las pruebas de HAI e IFI. Se utilizó el kit comercial "Chagatest HAI" de Laboratorios Wiener (Rosario, Argentina), según el protocolo recomendado por los fabricantes. Las muestras que resultaron positivas en la dilución 1:16 fueron consideradas positivas y se volvieron a analizar con diluciones seriadas (1:16 hasta 1:4096), para determinar el título final de cada una en la HAI (Villegas 2008).

Para la IFI, se prepararon las láminas con el antígeno en el Laboratorio de Zoonosis de la Escuela de Medicina Veterinaria de la Universidad Nacional. Con este afán, se tomó un cultivo de epimastigotos 
de T. cruzi en fase exponencial de crecimiento, en medio líquido de LIT (infusión de hígado y triptona). Se colocó $25 \mu$ l en cada uno de los pocillos de las láminas de IFI, en una concentración de $1.3 \times 10^{6}$ parásitos por ml en solución de Locke. Las láminas se dejaron secar en la estufa a $37^{\circ} \mathrm{C}$ durante 15 minutos, se fijaron con acetona pura por 2 minutos y se conservaron a $-20^{\circ} \mathrm{C}$ hasta su procesamiento.

Para realizar la IFI, se diluyeron los sueros 1:32 en buffer fosfato salino (PBS) y se colocaron $25 \mu \mathrm{l}$, en forma individual, en los pocillos de las láminas. En cada lámina, se colocaron, además, un suero control positivo y uno negativo. Las láminas se incubaron por 30 minutos a $37^{\circ} \mathrm{C}$ en una cámara húmeda; seguidamente, se lavaron 2 veces con PBS por 10 minutos y se agregó el conjugado (1:40 anti-IgG perro-FITC, MP Biomedicals®). Se volvieron a incubar por 30 minutos a $37^{\circ} \mathrm{C}$ en la cámara húmeda, se realizaron otros 2 lavados con PBS y se observó las láminas en microscopio de inmunofluorescencia. Los sueros que mostraron fluorescencia en la dilución 1:32 se consideraron positivos y se sometieron, nuevamente, a la IFI (diluciones 1:32 hasta 1:4096), para determinar el título final (Villegas 2008).

\section{Resultados}

Del total de 427 sueros caninos analizados, resultaron positivos en HAI e IFI 5, lo cual determina una seroprevalencia de $1.17 \%$ en los 28 cantones estudiados.

Los títulos de los sueros positivos en HAI e IFI, la provincia y el cantón en el que vivían los caninos seropositivos, así como información sobre reportes previos de casos de tripanosomiasis en caninos o reportes previos de la presencia del vector en el cantón se presentan en el Cuadro 1.

Cuadro 1. Títulos de anticuerpos y procedencia de los caninos seropositivos de T. cruzi

\begin{tabular}{|c|c|c|c|c|c|c|}
\hline \multirow[b]{2}{*}{ Canino } & \multirow{2}{*}{$\begin{array}{l}\text { HAI } \\
\text { Título }\end{array}$} & \multirow{2}{*}{$\begin{array}{c}\text { IFI } \\
\text { Título }\end{array}$} & \multirow[b]{2}{*}{ Provincia } & \multirow[b]{2}{*}{ Cantón } & \multicolumn{2}{|c|}{ Reportes previos de } \\
\hline & & & & & $\begin{array}{l}\text { vectores } \\
\text { en el cantón }\end{array}$ & $\begin{array}{c}\text { caninos con } \\
\text { T. cruzi }\end{array}$ \\
\hline 1 & $+(1: 1024)$ & $+(1: 1024)$ & Heredia & San Rafael & $\mathrm{Si}^{1}$ & $\mathrm{Si}^{\prime 2,3}$ \\
\hline 2 & $+(1: 1024)$ & $+(1: 512)$ & San José & Mora & $\mathrm{Sí}^{1}$ & No \\
\hline 3 & $+(1: 256)$ & $+(1: 256)$ & San José & Mora & $S 1^{1}$ & No \\
\hline 4 & $+(1: 1024)$ & $+(1: 1024)$ & Puntarenas & Golfito & No & No \\
\hline 5 & $+(1: 128)$ & $+(1: 128)$ & Guanacaste & Liberia & $S_{1}^{1}$ & No \\
\hline
\end{tabular}

${ }^{1}$ Zeledón 1981; ${ }^{2}$ Montenegro et al. 2002; ${ }^{3}$ Lizundia et al. 2014

Las características y hallazgos en la revisión clínica de los 5 caninos positivos en HAI e IFI de T. cruzi se muestran en el Cuadro 2. En el momento de la revisión clínica, los 5 caninos positivos se encontraban alerta, eran dóciles y presentaron temperatura, frecuencia cardiaca, frecuencia respiratoria, sonidos cardíacos y llenado capilar entre los rangos normales. Ninguno de estos caninos presentó, el día de esa revisión clínica, signos compatibles con la infección por T. cruzi, por ejemplo, tos, ascitis, intolerancia al 
ejercicio, letargia, desmayos, arritmias o dificultad respiratoria. Tampoco los propietarios observaron dichos signos clínicos en sus animales, con anterioridad.

Cuadro 2. Características de los 5 caninos seropositivos para T. cruzi

\begin{tabular}{cccccccc}
\hline Canino & Raza & Sexo & $\begin{array}{c}\text { Edad } \\
\text { (años) }\end{array}$ & Talla & $\begin{array}{c}\text { Membranas } \\
\text { mucosas }\end{array}$ & $\begin{array}{c}\text { Condición } \\
\text { corporal }\end{array}$ & $\begin{array}{c}\text { Permanencia fuera } \\
\text { o dentro del hogar }\end{array}$ \\
\hline 1 & SRD & Hembra & 1 & Mediano & Muy pálidas & Buena & Fuera \\
2 & French poodle & Hembra & 0.5 & Pequeño & Rosadas & Buena & Dentro \\
3 & French poodle & Macho & 1 & Pequeño & Rosadas & Buena & Dentro \\
4 & SRD & Macho & 4 & Mediano & Pálidas & Regular & Fuera \\
5 & SRD & Macho & 5 & Pequeño & Rosadas & Buena & Fuera \\
\hline
\end{tabular}

${ }^{*}$ SRD: Sin raza definida.

En 4 (14.3\%) del total de 28 cantones analizados, se determinaron perros seropositivos de T. cruzi. En 3 cantones (Golfito, Mora y Liberia), se encontraron perros seropositivos por primera vez en Costa Rica, mientras que en 1 (San Rafael de Heredia) ya se habían reportado perros seropositivos en otros estudios. Un total de 24 cantones resultó negativo en este estudio, de los cuales 4 (Aserrí, Santa Ana, Heredia Centro y Alajuela Centro) tenían reportes previos de caninos seropositivos de T. cruzi y en 20 cantones nunca se había buscado infecciones de T. cruzi en caninos (Cuadro 3 y Figura 1). Actualizando los datos, se han identificado, hasta la fecha, perros seropositivos en 15 cantones, mientras que en 20 no se han encontrado caninos con tripanosomiasis, y en 47 cantones aún no se han realizado estudios serológicos o no se han detectado canes seropositivos de T. cruzi (Cuadro 3 y Figura 1). 
Rev. Ciencias Veterinarias, Vol. 36, N² 2, [1-14], E-ISSN: 2215-4507, julio-diciembre, 2018 DOI: http://dx.doi.org/10.15359/rcv.36-2.1

URL: http://www.revistas.una.ac.cr/index.php/veterinaria/index

Cuadro 3. Cantones con estudios serológicos de T. cruzi en caninos de Costa Rica

\begin{tabular}{|c|c|c|c|}
\hline Provincia & Cantón & En estudios previos & En este estudio \\
\hline San José & San José & ND & Negativo \\
\hline San José & Escazú & ND & Negativo \\
\hline San José & Desamparados & ND & Negativo \\
\hline San José & Aserrí & POSITIVO $^{1,2}$ & Negativo \\
\hline San José & Mora & ND & POSITIVO \\
\hline San José & Santa Ana & POSITIVO $^{3}$ & Negativo \\
\hline San José & Alajuelita & ND & Negativo \\
\hline San José & Vásquez de Coronado & ND & Negativo \\
\hline San José & Tibás & ND & Negativo \\
\hline San José & Moravia & ND & Negativo \\
\hline San José & Montes de Oca & ND & Negativo \\
\hline San José & Curridabat & $\mathrm{ND}$ & Negativo \\
\hline Heredia & Heredia & POSITIVO $^{3}$ & Negativo \\
\hline Heredia & Barva & POSITIVO $^{2}$ & $\mathrm{NA}$ \\
\hline Heredia & Santo Domingo & ND & Negativo \\
\hline Heredia & San Rafael & POSITIVO $^{2,3}$ & POSITIVO \\
\hline Heredia & San Isidro & POSITIVO $^{3}$ & NA \\
\hline Heredia & Belén & POSITIVO $^{4}$ & $\mathrm{NA}$ \\
\hline Heredia & San Pablo & ND & Negativo \\
\hline Alajuela & Alajuela & POSITIVO $^{2}$ & Negativo \\
\hline Alajuela & Atenas & POSITIVO $^{2}$ & $\mathrm{NA}$ \\
\hline Alajuela & Poás & POSITIVO $^{3}$ & NA \\
\hline Alajuela & San Carlos & ND & Negativo \\
\hline Cartago & Cartago & ND & Negativo \\
\hline Cartago & La Unión & ND & Negativo \\
\hline Cartago & Oreamuno & ND & Negativo \\
\hline Cartago & El Guarco & ND & Negativo \\
\hline Guanacaste & Liberia & ND & POSITIVO \\
\hline Guanacaste & Santa Cruz & POSITIVO $^{1}$ & $\mathrm{NA}$ \\
\hline Guanacaste & Cañas & ND & Negativo \\
\hline Puntarenas & Esparza & POSITIVO $^{2}$ & NA \\
\hline Puntarenas & Golfito & ND & POSITIVO \\
\hline Puntarenas & Garabito & ND & Negativo \\
\hline Limón & Limón & ND & Negativo \\
\hline Limón & Pococí & ND & Negativo \\
\hline
\end{tabular}

ND: No hay dato. NA: No analizado

${ }^{1}$ Villegas 2008; ${ }^{2}$ Lizundia et al. 2014; ${ }^{3}$ Montenegro et al. 2002; ${ }^{4}$ Berrocal-Avila et al. 1993 


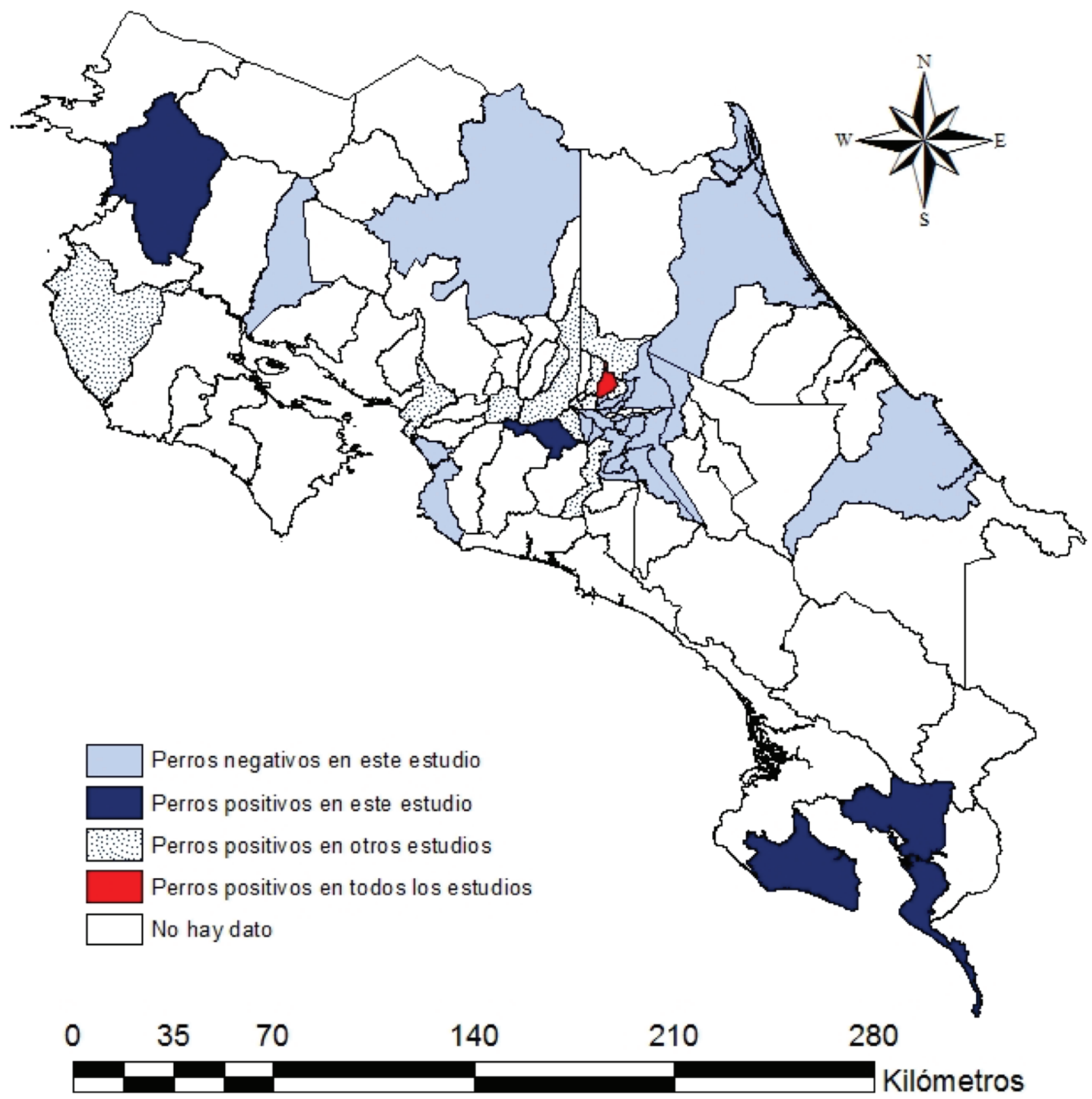

Figura 1. Reporte de estudios serológicos de T. cruzi en caninos de Costa Rica, 1993-2017. 


\section{Discusión}

La enfermedad de Chagas en Costa Rica no se consideraba un problema prioritario de salud pública hasta el 2003, cuando se inicia con el tamizaje del $100 \%$ de las donaciones sanguíneas (Norma de atención integral de la enfermedad de Chagas 2012; Campos-Fuentes \& Calvo-Fonseca 2013). En el 2005, la OMS declaró la enfermedad de Chagas como una enfermedad tropical desatendida, con el fin de implementar medidas de control. Actualmente, las infecciones por T. cruzi en humanos son de reporte obligatorio en Costa Rica y la vigilancia epidemiológica de los casos se realiza en forma pasiva (Norma de atención integral de la enfermedad de Chagas 2012). En contraste, este padecimiento no es de reporte obligatorio en animales ni tampoco existen programas para su vigilancia epidemiológica en nuestro país (Ministerio de Agricultura y Ganadería 2008). Es por esto que es importante continuar con la ejecución de investigaciones de tal enfermedad zoonótica en el país.

La seroprevalencia de T. cruzi en perros, en este estudio, resultó más baja en comparación con las reportadas en localidades, en investigaciones anteriores (Montenegro et al. 2002; Villegas 2008; Lizundia et al. 2014). Sin embargo, esos estudios fueron realizados en sitios donde se conocía la presencia de vectores o de tripanosomiasis canina, lo que podría ser la razón de esa diferencia en la seroprevalencia.

Actualmente, se han identificado perros seropositivos en 15 cantones de Costa Rica y se desconoce la presencia de casos de tripanosomiasis canina en 47 cantones del país; de estos cantones, en 22 se ha reportado con anterioridad la presencia de T. dimidiata (Zeledón 1981; Instituto Nacional de Biodiversidad 2017).

Aunque T. dimidiata es el principal vector de la tripanosomiasis americana en Costa Rica y tiene una alta predilección por sangre canina para su alimentación, parecen infectarse solamente un bajo número de triatominos, al alimentarse con sangre canina. Lo anterior demuestra una más baja infectividad (11.3 \%) de caninos que animales silvestres, como los didélfidos, en los cuales se encontró una infectividad del $44 \%$ (Calderón-Arguedas et al. 2001). En un estudio realizado en Costa Rica en el 2002, solamente un $5.5 \%$ de los perros analizados se diagnosticó como positivo de T. cruzi, mediante xenodiagnóstico, mientras que un $27.7 \%$ de estos caninos mostró anticuerpos contra el parásito (Montenegro et al. 2002), coincidiendo con lo que reporta la literatura en cuanto a que la mayoría de perros seropositivos representan un riesgo bajo de infección para los triatominos. No obstante, el hallazgo de caninos seropositivos de T. cruzi sugiere la presencia de vectores infectados en la localidad, por lo que se deben realizar más estudios para corroborar que esos sitios son endémicos y que puede existir un posible riesgo de infección tanto para los humanos como para los caninos.

La detección de anticuerpos contra T. cruzi en los perros indica tanto exposición al agente como infección de los caninos con el parásito (Tenney et al. 2014). Para el diagnóstico de infecciones de T. cruzi, se recomienda utilizar pruebas serológicas, ya que existe mayor probabilidad de detectar los anticuerpos en la sangre que en el parásito. Los anticuerpos se producirán durante toda la vida de los caninos y se descubrirán en las muestras de sangre, puesto que no hay tratamiento que elimine la infección en perros (Tenney et al. 2014).

En contraste, la parasitemia fluctúa en los caninos, aunque por lo general es muy baja, especialmente en la fase crónica, lo cual hace difícil el diagnóstico de animales infectados, mediante técnicas directas 
(microscopía de luz, cultivo, pruebas moleculares, xenodiagnósitco) (Eloy \& Lucheis 2009).

La razón por la cual no se detectó signos clínicos compatibles con la tripanosomiasis en los caninos seropositivos, probablemente, se debe a que estos se encontraban en la fase intermedia de la enfermedad, la cual se ha reportado que ocurre de manera asintomática; donde los parásitos se replican en las células musculares y al no haber aún gran daño, no se evidencia la sintomatología cardiaca. Sin embargo, en el transcurso de la infección, los individuos manifestarán un mayor grado de la sintomatología clínica (Tenney et al. 2014; Meyers et al. 2017).

En esta investigación, 2 caninos mostraron mucosas pálidas o muy pálidas. En ellos se encontraron garrapatas el día de la revisión clínica, lo cual pudo haber sido la razón del hallazgo clínico o, también, debido a alguna otra infección concomitante producida por un hemoparásito transmitido por garrapatas (Barrantes-González et al. 2016b).

Una limitante del estudio fue el no contar con la información sobre la existencia del vector en la vivienda de los caninos, así como otros datos tales como el tipo de construcción de esta, su cercanía a cafetales o lotes baldíos, la presencia de materiales acumulados en el área intra- o peridomiciliar, entre otros, los cuales se consideran como factores de riesgo de la tripanosomiasis (Norma de atención integral de la enfermedad de Chagas 2012). Esta información hubiera sido útil para relacionar las infecciones en los caninos con dichos factores de riesgo. Sin embargo, es importante resaltar que, en 3 de los cantones, en donde se reportaron por primera vez caninos seropositivos, se había reportado la presencia del vector (Mora, San Rafael y Liberia) (Zeledón 1981; Instituto Nacional de Biodiversidad 2017).

En el cantón de San Rafael de Heredia, se había reportado la presencia de vectores triatominos infectados con el parásito T. cruzi desde 1952 (Berrocal-Ávila et al. 1993). En 1993 se reportó el primer caso de tripanosomiasis aguda en un cachorro de 9 meses, el cual murió súbitamente y la necropsia estableció alteraciones cardiacas, confirmando T. cruzi como la causa de muerte. Además, en la vivienda del cachorro se encontraron vectores positivos (Berrocal-Ávila et al. 1993). Otros 2 estudios serológicos también encontraron caninos positivos en este cantón (Montenegro et al. 2002; Lizundia et al. 2014). Estos reportes sugieren que el cantón continúa reuniendo las condiciones que favorecen el establecimiento de las infecciones por T. cruzi en caninos.

Golfito fue el único cantón de Costa Rica en el que hasta el momento no se habían reportado vectores triatominos, pero se determinó un canino seropositivo. Esto puede deberse ya sea porque el vector está presente en el cantón, pero que no se ha investigado y encontrado hasta la fecha, o porque el canino se haya infectado en otra zona y luego llegara a residir al cantón de Golfito. El hecho no pudo ser investigado, ya que el día de la toma de la muestra los propietarios no indicaron cuánto tiempo llevaba el canino conviviendo con ellos en Golfito. Sin embargo, los dueños contaron que el perro vivía la mayor parte del tiempo afuera y en los alrededores de la casa, por lo que, en caso de encontrarse el vector en la zona, habría tenido la oportunidad de ingerirlo accidentalmente (Greene 2012; Berrizbeitia et al. 2013; Rodrigues 2015).

Los animales detectados como seropositivos en el presente trabajo fueron, sobre todo, animales jóvenes, lo cual concuerda con lo reportado en la literatura: que las infecciones por T. cruzi se dan, primordialmente, en perros jóvenes, ya sea porque los cachorros fueron infectados transplacentariamente, o bien porque 
ingirieron los triatominos por accidente al jugar con ellos (Greene 2012; Berrizbeitia et al. 2013; Rodrigues 2015). El cachorro y los caninos de 1 año de edad seropositivos de T. cruzi en este estudio probablemente tuvieron una infección reciente y, por lo tanto, existe la posibilidad de haberse infectado en el sitio de muestreo. Por el contrario, no se puede afirmar que los caninos de 4 y 5 años de edad que resultaron seropositivos para T. cruzi, en este estudio, tuvieran una infección reciente; tampoco es posible descartar que fueran trasladados al sitio de muestreo proviniendo infectados de otra localidad.

En esta investigación se reporta, por primera vez, la presencia de perros seropositivos en los cantones de Mora, Liberia y Golfito, donde se desconocía la presencia de tripanosomiasis canina. El hallazgo de perros seropositivos en 3 cantones adicionales es importante, ya que estos caninos se comportan como indicadores de la presencia de vectores infectados en la localidad, por lo que los resultados deben ser comunicados a los veterinarios y a las autoridades de salud pública, para que se tomen las medidas de control y prevención necesarias. La tripanosomiasis canina es de difícil diagnóstico clínico por la frecuente inespecificidad de sus síntomas y el diagnóstico a veces se realiza post mortem (Berrocal-Ávila et al. 1993). Los cuadros clínicos se confunden con miocarditis, arritmias o insuficiencias cardiacas de otras causas, por lo que los veterinarios deben hacer este diagnóstico diferencial. Las pruebas serológicas facilitan la identificación de los casos y sería importante continuar con estudios clínicos y epidemiológicos en caninos de nuestro país, de modo que sean considerados como parte de la Norma de atención integral de la enfermedad de Chagas.

\section{Conclusiones}

Se estableció una seroprevalencia baja (1.17 \%) en 28 cantones de Costa Rica y el presente estudio generó información importante sobre la presencia del agente en caninos de cantones donde no se habían reportado casos de tripanosomiasis o vectores. Son necesarias más investigaciones en caninos, sobre todo sistemáticas y en el nivel de comunidades y cantones, para establecer la distribución de las infecciones por T. cruzi en canes de Costa Rica. Por ser esta una enfermedad zoonótica y los perros considerados principales hospedadores domésticos de ella, además infectados en condiciones naturales más frecuentemente que los humanos, los resultados de estudios con caninos podrían utilizarse para estimar el riesgo de infecciones en humanos.

\section{Agradecimientos}

Los autores desean agradecer a todos los propietarios de caninos que estuvieron anuentes a participar en el proyecto de investigación, así como a los estudiantes y asistentes de la Escuela de Medicina Veterinaria de la Universidad Nacional, quienes colaboraron en las diferentes etapas de la indagación. Este proyecto fue financiado por fondos FIDA de la Fundación Universidad Nacional (FUNDAUNA).

\section{Referencias}

Arce-Fonseca, M., Carrillo-Sánchez, S. C., Molina-Barrios, R. M., Martínez-Cruz, M., Cedillo-Cobián, J. R., Henao-Díaz, Y. A. \& Rodríguez-Morales, O. 2017. Seropositivity for Trypanosoma cruzi in domestic dogs from Sonora, Mexico. Infect Dis Poverty 6(1): 120-127. Doi: 10.1186/s40249-017-0333-Z 
Barbabosa-Pliego, A., Gil, P. C., Hernández, D. O, Aparicio-Burgos, J. E., Montes de Oca-Jiménez, R., Martínez-Castañeda, J. S., Ochoa-García, L., Guzmán-Bracho, C., Estrada-Franco, J. G, Garg, N. J. \& Chagoyán, J. C. 2011. Prevalence of Trypanosoma cruzi in dogs (Canis familiaris) and triatomines during 2008 in a sanitary region of the State of Mexico, Mexico. Vector-Borne Zoonot 11(2): 151-156. Doi: 10.1089/vbz.2009.0163

Barrantes-González, A. V., Jiménez-Rocha, A. E., Romero-Zuñiga, J. J. \& Dolz, G. 2016a. Serology, molecular detection and risk factors of Ehrlichia canis infection in dogs in Costa Rica. Ticks Tick Borne Dis 7(6): 1245-1251. Doi: 10.1016/j.ttbdis.2016.07.006

Barrantes-González, A. V., Jiménez-Rocha, A. E., Romero-Zuñiga, J. J. \& Dolz, G. 2016b. Understanding Ehrlichia canis Infections in Dogs of Costa Rica: Hematological Findings and Indicative Clinical Signs. Open J. Vet. Med. 6(11): 163-175. Doi: 10.4236/ojvm.2016.611020

Berrizbeitia, M., Concepción, J. L., Carzola, V., Rodríguez, J., Cáceres, A. \& Quiñones, W. 2013. Seroprevalence of T. cruzi infection in Canis familiaris, state of Sucre, Venezuela. Biomédica 33(2): 214-225. Doi: doi.org/10.7705/biomedica.v33i2.7

Berrocal-Ávila, A., Morales-Acuña, J. A., Cordero, V. \& Villalobos, C. 1993. Miocarditis aguda chagásica en caninos de Costa Rica. Acute myocarditis due to Trypanosoma cruzi in dogs in Costa Rica. Revista de Ciencias Veterinarias 15(1): 51-59.

Calderón-Arguedas, O., Chinchilla, M., García, F. \& Vargas, M. 2001. Preferencias alimentarias de Triatoma dimidiata (Hemiptera: Reduviidae) procedente de la meseta central de Costa Rica a finales del siglo XX. Parasitología al día 25(3-4): 78-81. Doi: 10.4067/S0716-07202001000300002

Campos-Fuentes, E. \& Calvo-Fonseca, N. 2013. Confirmación diagnóstica del tamizaje de enfermedad de Chagas en Costa Rica. Rev. Costarr. Salud Pública 22(1): 4-8.

Castillo-Neyra, R., Chou Chu, L., Quispe-Machaca, V., Ancca-Juárez, J., Málaga Chavez, F. S., Bastos Mazuelos, M., Naquira, C., Bern, C., Gilman, R. H. \& Levy, M. Z. 2015. The potential of canine sentinels for reemerging Trypanosoma cruzi transmission. Prev. Vet. Med. 120(3-4): 349-356. Doi: $10.1016 /$ j.prevetmed.2015.04.014

Dias-Pérez, T., Borges-Figueiredo, F., Mendes, A. A., Laurentino-Silva, V., Madeira, M. D. F., PecanhaBrazil, R. \& Rodrigues-Coura, J. 2016. Prevalence of American trypanosomiasis and leishmaniases in domestic dogs in a rural area of the municipality of São João do Piauí, Piauí state, Brazil. Rev. Inst. Med. Trop. Sao. Paulo. 58(79): 1-6. Doi: 10.1590/S1678-9946201658079

Eloy, L. J. \& Lucheis, S. B. 2009. Canine trypanosomiasis: etiology of infection and implications for public health. J. Venom. Anim. Toxins Incl. Trop. Dis. 15(4): 589-611. Doi: 10.1590/s167891992009000400002

FUPROVI (Fundación Promotora de Vivienda). 2012. Situación de Vivienda y Desarrollo Urbano en Costa Rica en el 2012. Unidad de Investigación (UNIN).

Galvão, C. \& Justi, S. A. 2015. An overview on the ecology of Triatominae (Hemiptera: Reduviidae). Acta Trop. 151: 116-125. Doi: 10.1016/j.actatropica.2015.06.006 
Greene, C. 2012. Infectious diseases of the dog and cat. 4 ed. United States, Elsevier, p. 722-730.

INEC (Instituto Nacional de Estadística y Censos). 2012. Encuesta Nacional de Hogares, Julio 2012, Resultados Generales. Vol. 1, Año 3.

Instituto Nacional de Biodiversidad (INBio). 2017. Base de datos de especímenes (Atta), ficha de especies [en línea]: Triatoma dimidiata, especímenes y obvervaciones. http://www.crbio.cr:8080/ neoportal-web/?q=triatoma\%20dimidiata (consultado el 1 de mayo, 2017).

List, M. 2009. Situación actual de las poblaciones caninas en Costa Rica. Servicio Nacional de Salud Animal, Costa Rica. Dic. 2009.

Lizundia, R., Picado, A., Cordero, M., Calderón, A., Deborggraeve, S., Montenegro, V. M. \& Urbina, A. 2014. Molecular and serological rapid tests as markers of Trypanosoma cruzi infection in dogs in Costa Rica. Tropical Parasitology 4(2): 111-114. Doi: 10.4103/2229-5070.138539

Matamoros, X. 2002. Caracterización por Biodemas y Zimodemas de cepas de Trypanosoma cruzi, circulantes en zonas endémicas de las Repúblicas de Nicaragua y Costa Rica. Tesis de maestría en Enfermedades Tropicales, para optar por el título de Magister Scientiae. Universidad Nacional. Costa Rica.

Meyers, A.C., Meinders, M. \& Hamer, S. A. 2017. Widespread Trypanosoma cruzi infection in government working dogs along the Texas-Mexico border: Discordant serology, parasite genotyping and associated vectors. PLoS Negl. Trop. Dis. 11(8): e0005819. Doi: 10.1371/journal.pntd.0005819

Ministerio de Agricultura y Ganadería (MAG). 2008. Listado de enfermedades animales de declaración obligatoria. Decreto No. 34669-MAG. Presidencia de la República y Ministerio de Agricultura y Ganadería, Costa Rica.

Ministerio de Salud de Costa Rica. 2015. Memoria Institucional 2014. San José, Costa Rica, El Ministerio.

Montenegro, V. M., Jiménez, M., Dias, J. C. \& Zeledón, R. 2002. Chagas disease in dogs from endemic areas of Costa Rica. Mem. Inst. Oswaldo Cruz 97(4): 491囚494. Doi: 10.1590/S007402762002000400006

Norma de atención integral de la enfermedad de Chagas. 2012. Decreto Ejecutivo N. ${ }^{\circ}$ 37269-S. Presidencia de la República y Ministerio de Salud. Gobierno de Costa Rica.

Nieto, P. D., Boughton, R., Dorn, P. L., Steurer, F., Raychaudhuri, S., Esfandiari, J., Gonçalves, E., Díaz, J. \& Malone, J. B. 2009. Comparison of two immunochromatographic assays and the indirect immunofluorscence antibody test for diagnosis of Trypanosoma cruzi infection in dogs in south central Louisiana. Vet. Parasitol. 165(3-4): 241-247. Doi: 10.1016/j.vetpar.2009.07.010

Organización Mundial de la Salud (OMS). 2017. Nota descriptiva [en línea]: La enfermedad de Chagas (tripanosomiasis americana). http://www.who.int/mediacentre/factsheets/fs340/es// (consultado el 20 de enero, 2018).

Pérez, F., Vargas, L. G., Fernández, O. \& Velásquez, R. 1984. Enfermedad de chagas aguda: reporte de tres casos tratados con Nifurtimox. Acta Méd. Costarric. 27: 94-101. 
Rodrigues, J. 2015. The main sceneries of Chagas disease transmission. The vectors, blood and oral transmissions - A comprehensive review. Mem. Inst. Oswaldo Cruz 110(3): 277-282.

Saldaña, A., Calzada, J. E., Pineda, V., Perea, M., Rigg, C., González, K., Santamaría, A. M., Gottdenker, N. L. \& Chaves, L. F. 2015. Risk factors associated with Trypanosoma cruzi exposure in domestic dogs from a rural community in Panama. Mem. Inst. Oswaldo Cruz 110(7): 936-944. Doi: 10.1590/0074-02760150284

Sociedad Mundial para la Protección Animal. 2012. Situación canina en los hogares de la Gran Área Metropolitana. Costa Rica. Dic. 2012.

Tenney, T. D., Curtis-Robles, R., Snowden, K. F. \& Hamer, S. A. 2014. Shelter dogs as sentinels for Trypanosoma cruzi transmission across Texas. Emerg. Infect. Dis. 20(8): 1323-1326. Doi: 10.3201/ eid2008.131843

Villegas, A. 2008. Seroprevalencia de la infección por Trypanosoma cruzi en perros de dos zonas rurales con altos y bajos índices de infestación por Triatoma dimidiata. Tesis para optar por el grado académico de Licenciatura en Medicina Veterinaria. Universidad Nacional. Costa Rica.

Zeledón, R. 1981. El Triatoma dimidiata y su relación con la enfermedad de Chagas. Editorial Universidad Estatal a Distancia. San José, Costa Rica, p. 9, 36-47.

Zeledón, R., Hanson, P. \& Zumbado, M. 2016. Guía de artrópodos de importancia médica y veterinaria. Editorial Universidad Estatal a Distancia. San José, Costa Rica, p. 13-18. 